\title{
Male current smokers have low awareness and optimistic bias about COPD: field survey results about COPD in Korea
}

This article was published in the following Dove Medical Press journal: International Journal of COPD

\section{Yong II Hwang, ${ }^{1,2}$ Yong Bum Park, ${ }^{2,3}$ Hyoung Kyu Yoon, ${ }^{4}$ Tae-Hyung Kim, ${ }^{5}$ Kwang Ha Yoo, ${ }^{6}$ Chin Kook Rhee, ${ }^{4}$ Joo Hun Park, 7 Seung Hun Jang, ${ }^{1,2}$ Sunghoon Park, ${ }^{1,2}$ Joo-Hee Kim, ${ }^{1,2}$ Jiyoung Park, ${ }^{1,2}$ Ki-Suck Jung ${ }^{1,2}$}

'Department of Pulmonary, Allergy and Critical Care Medicine, Hallym University Sacred Heart Hospital, Anyang-si, South Korea; ${ }^{2}$ Lung Research Institute of Hallym University College of Medicine, Chuncheon, South Korea; ${ }^{3}$ Department of Pulmonary, Allergy and Critical Care Medicine, Hallym University Kangdong Sacred Heart Hospital, Chuncheon, South Korea; ${ }^{4}$ Division of Pulmonology, Critical Care and Sleep Medicine, Department of Internal Medicine, College of Medicine, The Catholic University of Korea, Seoul, South Korea; ${ }^{5}$ Division of Pulmonology, Department of Internal Medicine, Hanyang University Guri Hospital, Hanyang University College of Medicine, Guri, South Korea; ${ }^{6}$ Department of Internal Medicine, Konkuk University School of Medicine, Seoul, South Korea; ${ }^{7}$ Department of Internal Medicine, Ajou University School of Medicine, Suwon, South Korea

Correspondence: Yong II Hwang Division of Pulmonary, Allergy and Critical Care Medicine, Department of Internal Medicine, Hallym University Sacred Heart Hospital, 22, Gwanpyeong-ro 170 Beon-gil, Dongan-gu, Anyang-si, Gyeonggi-do, South Korea $\mathrm{Tel}+82313803715$

Fax +82 31 3803973

Email hyicyk@hallym.or.kr
Background: Smoking is a major risk factor for COPD. However, there is low COPD awareness among smokers. We conducted a field survey to investigate COPD awareness, optimistic bias associated with COPD, and COPD prevalence (using handheld spirometry) among current male smokers.

Subjects and methods: We enrolled currently smoking males aged over 40 years, who completed a self-administered questionnaire. The questionnaire consisted of six parts: 1) baseline demographics, 2) participants' awareness of COPD and pulmonary function tests, 3 ) presence of COPD-related respiratory symptoms and experience with pulmonary function testing, 4) optimistic bias about COPD, 5) willingness to change attitude toward respiratory health, and 6) preference of media for obtaining health-related information. Pulmonary function was assessed via handheld spirometry by two experienced pulmonary function laboratory technicians after completion of the questionnaire.

Results: We enrolled 105 participants. Only 24.8\% knew of COPD. Awareness of pulmonary function testing was reported by $41.9 \%$ of participants, and $30.5 \%$ had previously undertaken pulmonary function tests. Among the subjects who had not previously undergone pulmonary function tests, $47 \%$ were not aware of their existence. The mean optimistic bias scores were 3.9 and 4.0, respectively, reflecting the general perception, among participants, that they were about as likely to develop COPD as similarly aged smokers and friends, respectively. A total of $40.0 \%$ of participants perceived personal COPD risk to be lower than COPD risk among their friends. Abnormal handheld spirometry results were observed in $28.6 \%$ of participants. Among the subjects with abnormal handheld spirometry results, $36.7 \%$ had $\mathrm{FEV}_{1}$ values $<50 \%$ of the predicted value.

Conclusion: In conclusion, current male smokers had poor awareness of COPD. Participants perceived their risk of developing COPD to be no higher than their friends' COPD risk. Strategies to increase COPD awareness among high-risk groups should be developed.

Keywords: COPD, awareness, optimistic bias, handheld spirometry

\section{Introduction}

COPD - currently the fourth leading cause of death in the world - is both preventable and treatable, but remains an important public health challenge. ${ }^{1}$ Unfortunately, COPD is well known to be underdiagnosed and undertreated. ${ }^{2-4}$ In Korea, the prevalence of COPD has not changed significantly since 2010, ranging from $13.1 \%$ to $14.6 \%$ since then. ${ }^{5}$ Furthermore, only $2.8 \%$ and $1.6 \%$ of COPD patients were estimated to be diagnosed and treated, respectively, in $2015 . .^{5}$ These proportions reflect a lack of improvement relative to previous prevalence studies conducted in Korea. ${ }^{4,6}$ 
While screening for COPD in asymptomatic adults is not recommended, ${ }^{7}$ active case-finding strategies should be considered for early diagnosis of COPD in high-risk subjects. ${ }^{8-11}$ Although smoking is the most commonly encountered risk factor for COPD, ${ }^{1} \mathrm{COPD}$ awareness among smokers is low in Korea. Among smokers participating in a smoking cessation program, only $1.0 \%$ knew of COPD, whereas $91.3 \%$ of them perceived that smoking is the cause of COPD. ${ }^{12}$

Interestingly, smokers are often confident about their health status, irrespective of the presence of respiratory symptoms; ${ }^{12,13}$ this phenomenon is known as "optimistic bias". Optimistic bias can be defined as the tendency for people to underrate risk when it pertains to themselves, compared with their view of risk among other people in the same conditions. ${ }^{14,15}$ Increasing the awareness of COPD among smokers has been suggested as an important step toward reducing the underdiagnosis and undertreatment of $\mathrm{COPD},{ }^{13}$ with the additional benefit of bolstering active case-finding efforts. ${ }^{8-11}$

We conducted a field survey to inform the development of a COPD awareness campaign for smokers. We report COPD awareness and optimistic bias levels, as well as handheld spirometry findings among current male smokers. Some of this work was presented as an abstract at an international conference. ${ }^{16}$

\section{Subjects and methods Study design and study population}

In this field survey, we randomly recruited participants at Seoul Station, which is a busy travel hub in Seoul, Korea, with a large floating population. We enrolled males aged 40 years and older who were current tobacco smokers at the time of enrollment. All participants provided written informed consent before participating. This study was approved by the institutional review board of Hallym University Sacred Heart Hospital (IRB no. 2018-12-001) and was conducted in accordance with the Declaration of Helsinki.

\section{Procedures}

All participants answered a structured questionnaire and underwent pulmonary function testing. The questionnaire consisted of six parts: 1) baseline demographics, 2) participants' awareness of COPD and pulmonary function tests, 3) presence of COPD-related respiratory symptoms and experience with pulmonary function testing, 4) optimistic bias about COPD, 5) willingness to change attitude toward respiratory health, and 6) preference of media for obtaining health-related information. The last two parts were conducted after pulmonary function testing was completed.

\section{Data collection and analysis}

Yes/no questions assessed awareness of COPD and pulmonary function tests, presence of respiratory symptoms, and experience with pulmonary function tests. Participants who had not previously undergone pulmonary function testing were asked to provide reasons for not having previously done so.

We assessed optimistic bias among participants using a 7-point scale developed by Weinstein. ${ }^{17,18}$ The relevant questions were worded as follows: "Compared to other men/women of my age or my friends, my chance to getting COPD in the future is: much below average, below average, a little below average, average, a little above average, above average, much above average." In analyzing optimistic bias, the seven possible responses were assigned numerical values ranging from 1 to 7 . Scores less than the median value (4 points) imply an optimistic bias.

Pulmonary function was assessed via handheld spirometry ${ }^{8}$ conducted by two experienced pulmonary function laboratory technicians after the first four parts of the questionnaire. Three repeats of the handheld spirometry were performed per participant. The highest values of $\mathrm{FEV}_{1}$ and $\mathrm{FEV}_{6}$ were recorded. The detailed procedure of handheld spirometry was followed according to the user manual provided by the manufacturer. ${ }^{19}$ The spirometry results were released to each participant immediately after the individual tests.

Willingness to change attitude was assessed by asking whether the participants would undergo pulmonary function tests, quit smoking, make efforts to avoid COPD, and look for information about COPD. A 5-point scale was used for willingness evaluation, ranging from 1 (not at all) to 5 (very much).

Finally, we investigated sources of health-related information. We asked the participants about their most preferred, most reliable, and most useful tools to get health information.

All interview data were collected and managed anonymously. Descriptive statistics (eg, frequencies and mean $\pm \mathrm{SD}$ ) were calculated for all study variables.

\section{Results}

\section{Baseline characteristics}

A total of 105 subjects were enrolled. The age distribution was as follows: $40-49$ years, $26.7 \%$; $50-59$ years, $34.3 \%$; 60-69 years, $32.4 \%$; and 70 years or older, $6.7 \%$. The mean smoking duration was 31.1 pack-years. Dyspnea was present in $28.6 \%$ of the participants (Table 1).

\section{Respiratory health awareness}

In response to the question, "Are you concerned about respiratory health?", $60 \%$ of the participants responded 
Table I Baseline characteristics

\begin{tabular}{l|l}
\hline Characteristics & Total $(\mathbf{N}=\mathbf{1 0 5})$ \\
\hline Male $(\mathrm{n}, \%)$ & $105(100)$ \\
\hline Age group, years $(\mathrm{n}, \%)$ & \\
$40-49$ & $28(26.7)$ \\
$50-59$ & $36(34.3)$ \\
$60-69$ & $34(32.4)$ \\
$\geq 70$ & $7(6.7)$ \\
\hline Smoking amount, pack-years (mean \pm SD) & $31.1 \pm 23.5$ \\
\hline Presence of respiratory symptoms $(\mathrm{n}, \%)$ & \\
$\quad$ Cough or sputum & $69(65.7)$ \\
$\quad$ Dyspnea & $30(28.6)$ \\
\hline Concern about respiratory health, yes $(\mathrm{n}, \%)$ & $63(60.0)$ \\
\hline
\end{tabular}

"yes". However, only $24.8 \%$ of the participants knew about COPD; $41.9 \%$ and $30.5 \%$ of the participants were aware of and had previously undergone pulmonary function testing, respectively (Table 2). Among the subjects who had not undergone pulmonary function testing, $47 \%$ were not aware of the existence of pulmonary function tests (Table 3 ).

\section{Optimistic bias}

The mean optimistic bias score was $3.9 \pm 1.75$ when participants were asked to compare personal health with that of similarly aged smokers. When participants were asked to compare their self-reported health status with that of their friends, the mean optimistic bias score was $4.0 \pm 1.76$ (Table 4; Figure 1).

\section{Handheld spirometry results}

When we regarded $\mathrm{FEV}_{1} / \mathrm{FEV}_{6} \leq 0.77$ as abnormal, ${ }^{8} 30$ participants (28.6\%) had abnormal spirometry results. Among the participants with abnormal spirometry results, 11 (36.7\%) had $\mathrm{FEV}_{1}$ values $<50 \%$ of the predicted value (Table 5).

\section{Change of attitude}

After receiving their handheld spirometry results, $60 \%$ of the participants responded that they would undergo further pulmonary function testing in the future and $45.7 \%$ of the participants reported that they would quit smoking (Figure 2).

Table 2 Insight about respiratory disease

\begin{tabular}{l|l}
\hline Variables & $\mathbf{n}(\%)$ \\
\hline Concern about respiratory health, yes & $63(60.0)$ \\
\hline Awareness of COPD, yes & $26(24.8)$ \\
\hline Awareness of spirometry, yes & $44(41.9)$ \\
\hline Experience of spirometry, yes & $32(30.5)$ \\
\hline
\end{tabular}

Table 3 Reasons for not taking pulmonary function test

\begin{tabular}{l|l}
\hline Answers & $\%$ \\
\hline I did not know pulmonary function test & 47 \\
\hline I knew pulmonary function test, but I did not need to do it & 37 \\
\hline Doctor did not recommend pulmonary function test & 7 \\
\hline Others & 5 \\
\hline No answer & 4 \\
\hline
\end{tabular}

\section{Preferred media}

The participants most commonly preferred television as the most reliable and useful form of medium to obtain health-related information. Doctors were the most preferred information source among $9.5 \%$ of the participants, the most reliable among $28.6 \%$ of the participants, and the most useful among $26.7 \%$ of the participants (Figure 3).

\section{Discussion}

In this field survey about COPD, we selected current male smokers at risk of developing COPD ${ }^{1}$ from a setting with a large floating population. We found that $63 \%$ of current male smokers were concerned about respiratory health. However, only one-quarter of current male smokers were aware of COPD, and $70 \%$ of them had not previously undertaken pulmonary function testing. The main reason for previously not having done pulmonary function tests was lack of awareness. Although the level of COPD awareness was higher than that observed in previous research that included participants of a smoking cessation program ${ }^{12}$ and a nation-wide telephone survey from Korea ${ }^{13}$ and Spain, ${ }^{20}$ a large proportion of our participants were not familiar with COPD or pulmonary function testing. This suggests that a program to raise awareness of COPD and pulmonary function testing should be provided to the high-risk population.

Optimistic bias is a cognitive bias that causes people to believe that they are less likely to experience negative events compared to others. This cognitive bias in risk perception may negatively influence health behavior and reinforce cigarette smoking over time. ${ }^{21}$ We demonstrated optimistic bias related to COPD among current male smokers. In our study, participants perceived that they were about as likely to develop COPD as similarly aged smokers and friends. However, $40.0 \%$ and $37.1 \%$ of them believed that their risk of COPD was less than the risk carried by their friends and other people of similar age, respectively (Table 4). There have been many studies about optimistic bias among smokers, and it is well known that smokers minimize the personal health risks of cigarette smoking. ${ }^{22}$ The majority of smokers 
Table 4 Optimistic bias

\begin{tabular}{l|l|l}
\hline & $\begin{array}{l}\text { Compared to other men/women of my age, } \\
\text { my chance to get COPD in the future is }\end{array}$ & $\begin{array}{l}\text { Compared to my friends, my chance to get } \\
\text { COPD in the future is }\end{array}$ \\
\hline Score (mean \pm SD) & $3.9 \pm 1.75$ & $4.0 \pm 1.75$ \\
\hline $\begin{array}{l}\text { Frequency }(\mathrm{n}, \%) \\
\text { Much below average }\end{array}$ & $10(9.5)$ & $12(11.5)$ \\
Below average & $21(20.0)$ & $22(21.2)$ \\
A little below average & $8(7.6)$ & $8(7.7)$ \\
Average & $23(21.9)$ & $13(12.5)$ \\
A little above average & $19(18.1)$ & $22(21.2)$ \\
Above average & $19(18.1)$ & $20(19.2)$ \\
Much above average & $5(4.8)$ & $7(6.7)$ \\
\hline
\end{tabular}

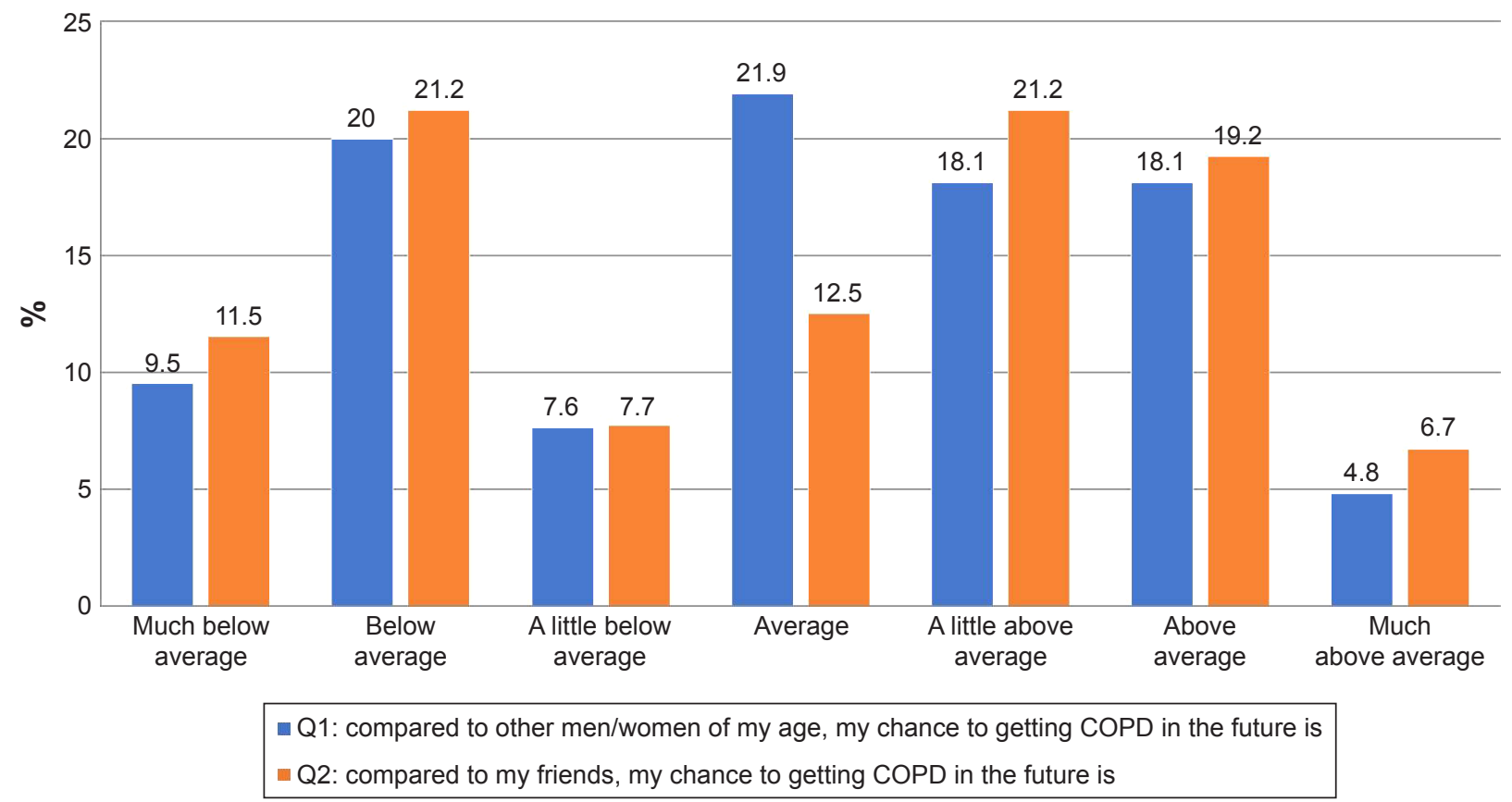

Figure I Distribution of optimistic bias.

Table 5 Distribution of handheld spirometry results

\begin{tabular}{|c|c|c|c|c|}
\hline & \multicolumn{4}{|c|}{ Age group (years) } \\
\hline & $40-49$ & $50-59$ & $60-69$ & $\geq 70$ \\
\hline \multicolumn{5}{|l|}{$\mathrm{FEV}_{1} / \mathrm{FEV}_{6} \leq 0.77(\mathrm{n}=30)$} \\
\hline $\mathrm{FEV}_{1} \geq 80 \%$ (n, \%) & $0(0)$ & $2(6.7)$ & I (3.3) & $0(0)$ \\
\hline $50 \leq \mathrm{FEV}_{1}<80(\mathrm{n}, \%)$ & $2(6.7)$ & $5(16.7)$ & $8(26.7)$ & I (3.3) \\
\hline $30 \leq \mathrm{FEV}_{1}<50(\mathrm{n}, \%)$ & $0(0)$ & $2(6.7)$ & $6(20.0)$ & $\mathrm{I}(3.3)$ \\
\hline $\mathrm{FEV}_{1}<30(\mathrm{n}, \%)$ & $0(0)$ & I (3.3) & $0(0)$ & I (3.3) \\
\hline Sub-total (n, \%) & $2(6.7)$ & $10(33.3)$ & $15(50.0)$ & $3(10.0)$ \\
\hline \multicolumn{5}{|l|}{$\mathrm{FEV}_{1} / \mathrm{FEV}_{6}>0.77(\mathrm{n}=75)$} \\
\hline $\mathrm{FEV}_{1} \geq 80 \%(\mathrm{n}, \%)$ & $19(25.3)$ & I5 (20.0) & $9(12.0)$ & I (I.3) \\
\hline $50 \leq \mathrm{FEV}_{1}<80(\mathrm{n}, \%)$ & $7(9.3)$ & II (I4.7) & $9(12.0)$ & $3(4.0)$ \\
\hline $30 \leq \mathrm{FEV}_{1}<50(\mathrm{n}, \%)$ & $0(0)$ & $0(0)$ & $\mathrm{I}(1.3)$ & $0(0)$ \\
\hline $\mathrm{FEV}_{1}<30(\mathrm{n}, \%)$ & $0(0)$ & $0(0)$ & $0(0)$ & $0(0)$ \\
\hline Sub-total (n, \%) & 26 (34.7) & 26 (34.7) & $19(25.3)$ & $4(5.3)$ \\
\hline
\end{tabular}




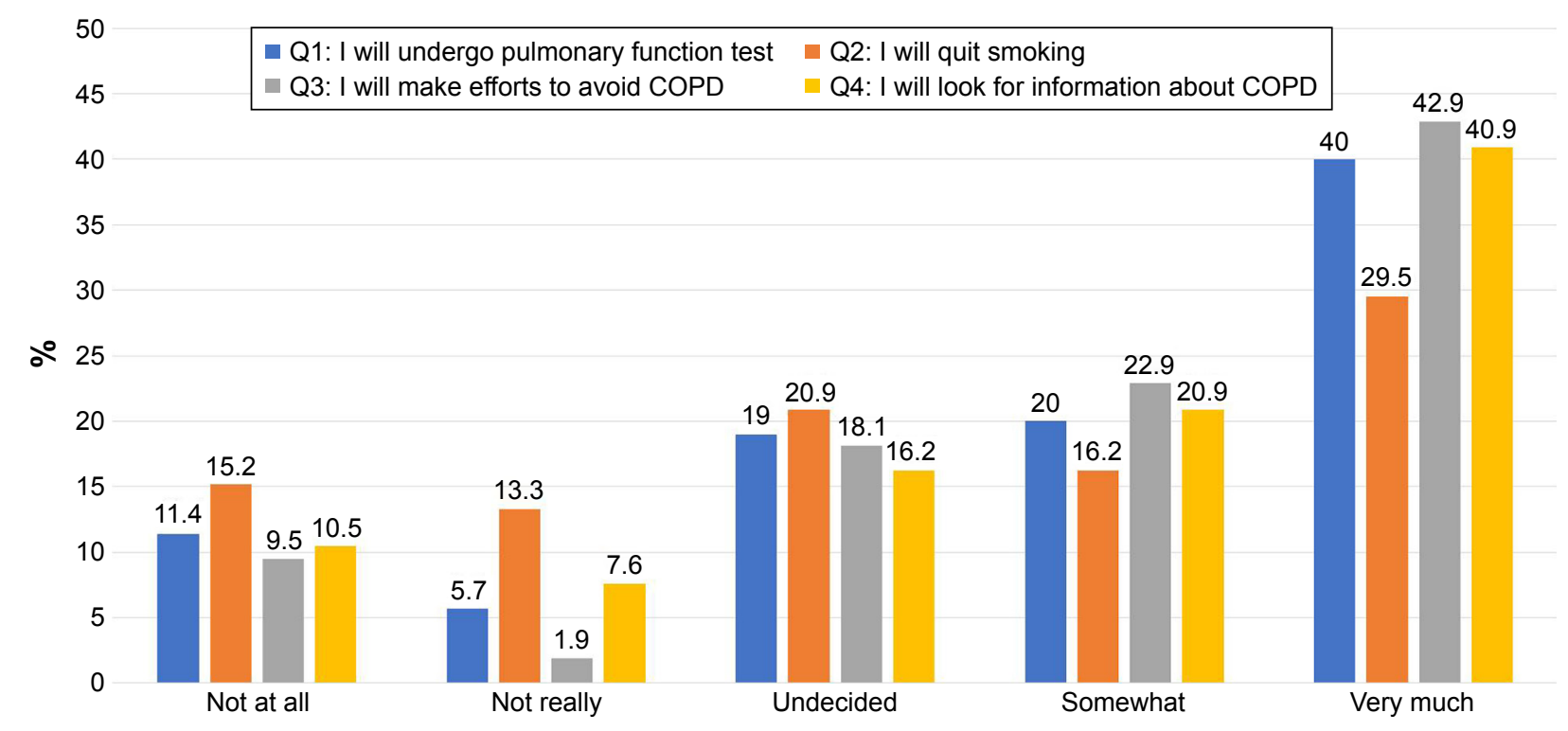

Figure 2 Willingness of attitude change.

underestimate their risk of smoking-related conditions compared with nonsmokers. ${ }^{23,24}$ However, to the best of our knowledge, this was the first study investigating optimistic bias among smokers, specifically as it relates to COPD.

We found that $28.6 \%$ of the participants had abnormal handheld spirometry results, which required further conventional spirometry to confirm COPD. ${ }^{8}$ This finding was consistent with our previous active COPD case-finding study, ${ }^{8}$ reinforcing the importance of active COPD case finding. However, the distribution of $\mathrm{FEV}_{1}$ was different from that of the active case-finding study, ${ }^{8}$ which had more participants with impaired pulmonary function.

Regarding willingness to change attitude about respiratory health and COPD, $60.0 \%$ and $45.7 \%$ of the participants responded that they would undergo further pulmonary function testing in the future and quit smoking, respectively. Also, $65.8 \%$ and $61.8 \%$ answered that they would make efforts to avoid COPD and look for the COPD-related information, respectively. These results point to their desire to avoid developing COPD. However, the proportion of participants intending to quit smoking was not different from that revealed by a nationwide COPD awareness survey. ${ }^{13}$ This suggests that a more personalized education program should be implemented to improve smoking cessation intent for COPD prevention.

In this study, television (which was preferred by $59 \%$ of the participants) was found to be the most preferred medium to obtain health-related information; $9.5 \%$ of the participants preferred receiving health information from

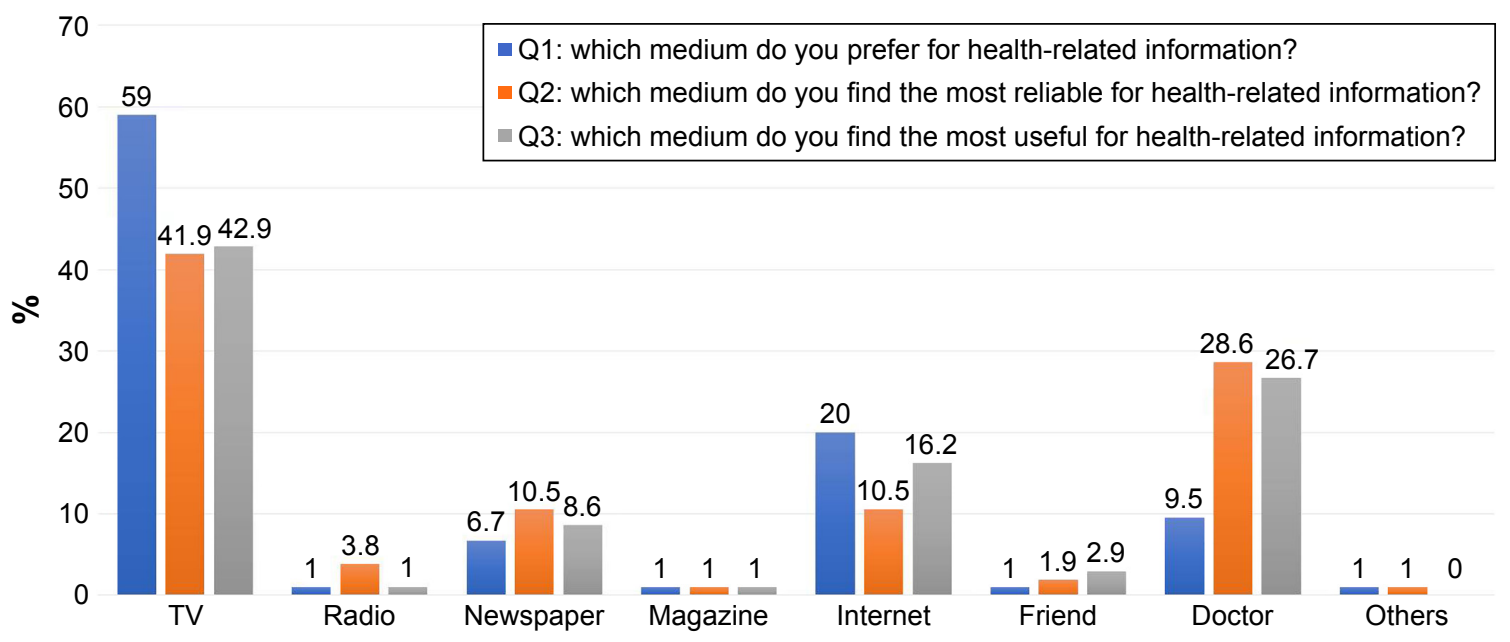

Figure 3 Preference of media for the health-related information. 
doctors. These proportions are similar to those found among participants of the previously mentioned smoking cessation program. ${ }^{12}$ Television was also perceived to be the most reliable (by $41.9 \%$ of the participants) and useful (by $42.9 \%$ of the participants) source of health-related information. Doctors were also considered a preferred source, with $28.6 \%$ of the participants identifying doctors as the most reliable information sources and $26.7 \%$ considering them the most useful.

Our study had several limitations. First, we only enrolled male participants, so the findings may not be applicable to female smokers. However, unlike the Western world, COPD is predominantly found in males in Korea, ${ }^{25,26}$ and we believe that our findings could reflect the current status in Korea. Second, our study was mainly qualitative; we could not analyze our field survey findings in detail. However, we think that the results of our study could provide basic data for developing a policy to increase public awareness of COPD in Korea.

\section{Conclusion}

This study showed that male smokers in Korea were concerned about their respiratory health and perceived that their risk of developing COPD was not high. About $30 \%$ of participants had dyspnea and abnormal pulmonary function. However, male smokers in Korea were not familiar with COPD, and many of them had no desire to change their attitude toward respiratory health and COPD prevention. We recommend the implementation of strategies to increase COPD awareness among high-risk groups.

\section{Acknowledgment}

This work was presented at the CHEST 2017 Annual Meeting and was published in CHEST, Volume 152, Issue 4, Supplement.

\section{Disclosure}

The authors report no conflicts of interest in this work.

\section{References}

1. Global Initiative for Chronic Obstructive Lung Disease. Global strategy for the diagnosis, management, and prevention of chronic obstructive pulmonary disease. Updated 2018 [Internet]. Bethesda, MD: Global Initiative for Chronic Obstructive Lung Disease; 2018. Available from: http://www.goldcopd.org. Accessed April 1, 2018.

2. Soriano JB, Zielinski J, Price D. Screening for and early detection of chronic obstructive pulmonary disease. Lancet. 2009;374(9691):721-732.

3. Bednarek M, Maciejewski J, Wozniak M, Kuca P, Zielinski J. Prevalence, severity and underdiagnosis of COPD in the primary care setting. Thorax. 2008;63(5):402-407.

4. Yoo KH, Kim YS, Sheen SS, et al. Prevalence of chronic obstructive pulmonary disease in Korea: the fourth Korean National Health and Nutrition Examination Survey, 2008. Respirology. 2011;16(4):659-665.
5. Hwang YI, Park YB, Yoo KH. Recent trends in the prevalence of chronic obstructive pulmonary disease in Korea. Tuberc Respir Dis (Seoul). 2017;80(3):226-229.

6. Hwang YI, Yoo KH, Sheen SS, et al. Prevalence of chronic obstructive pulmonary disease in Korea: the result of forth Korean National Health and Nutrition Examination Survey. Tuberc Respir Dis (Seoul). 2011; 71(5):328-334

7. US Preventive Services Task Force (USPSTF); Siu AL, BibbinsDomingo K, et al. Screening for chronic obstructive pulmonary disease: US Preventive Services Task Force recommendation statement. JAMA. 2016;315(13):1372-1377.

8. Kim JK, Lee CM, Park JY, et al. Active case finding strategy for chronic obstructive pulmonary disease with handheld spirometry. Medicine. 2016;95(50):e5683:50.

9. Lee SW, Yoo J-H, Park MJ, et al. Early diagnosis and management of chronic obstructive pulmonary disease. Tuberc Respir Dis (Seoul). 2011;70(4):293-300.

10. Lee JY, Rhee CK, Jung KS, Yoo KH. Strategies for management of the early chronic obstructive lung disease. Tuberc Respir Dis (Seoul). 2016;79(3):121-126.

11. Press VG, Cifu AS, White SR. Screening for chronic obstructive pulmonary disease. JAMA. 2017;318(17):1702-1703.

12. Seo JY, Hwang YI, Mun SY, et al. Awareness of COPD in a high risk Korean population. Yonsei Med J. 2015;56(2):362-367.

13. Mun SY, Hwang YI, Kim JH, et al. Awareness of chronic obstructive pulmonary disease in current smokers: a nationwide survey. Korean $J$ Intern Med. 2015;30(2):191-197.

14. Weinstein ND. Unrealistic optimism about susceptibility to health problems. J Behav Med. 1982;5(4):441-460.

15. Masiero M, Riva S, Oliveri S, Fioretti C, Pravettoni G. Optimistic bias in young adults for cancer, cardiovascular and respiratory diseases: a pilot study on smokers and drinkers. J Health Psychol. 2018;23(5):645-656.

16. Hwang YI, Park S, Kim JH, et al. Field survey result about COPD in current smokers: optimistic bias in current smokers. Chest. 2017;152(4): A795.

17. Weinstein ND. Why it won't happen to me: perceptions of risk factors and susceptibility. Health Psychol. 1984;3(5):431-457.

18. Weinstein ND. Unrealistic optimism about susceptibility to health problems: conclusions from a community-wide sample. J Behav Med. 1987;10(5):481-500.

19. Repiratory monitor COPD-6. User training manual. Ennis: Vitalograph (Ireland) Ltd; 2013. Available from: https://vitalograph.com/downloads/ view/15. Accessed January 8, 2019.

20. Soriano JB, Calle M, Montemayor T, Alvarez-Sala JL, Ruiz-Manzano J, Miravitlles M. The general public's knowledge of chronic obstructive pulmonary disease and its determinants: current situation and recent changes. Arch Bronconeumol. 2012;48(9):308-315.

21. Masiero M, Lucchiari C, Pravettoni G. Personal fable: optimistic bias in cigarette smokers. Int J High Risk Behav Addict. 2015;4(1):e20939.

22. Weinstein ND. Accuracy of smokers' risk perceptions. Ann Behav Med. 1998;20(2):135-140.

23. Ayanian JZ, Cleary PD. Perceived risks of heart disease and cancer among cigarette smokers. JAMA. 1999;281(11):1019-1021.

24. Strecher VJ, Kreuter MW, Kobrin SC. Do cigarette smokers have unrealistic perceptions of their heart attack, cancer, and stroke risks? J Behav Med. 1995;18(1):45-54.

25. Hwang YI, Lee SH, Yoo JH, et al. History of pneumonia is a strong risk factor for chronic obstructive pulmonary disease (COPD) exacerbation in South Korea: the Epidemiologic Review and Prospective Observation of COPD and Health in Korea (EPOCH) study. J Thorac Dis. 2015; 7(12):2203-2213.

26. Lee JY, Chon GR, Rhee CK, et al. Characteristics of patients with chronic obstructive pulmonary disease at the first visit to a pulmonary medical center in Korea: the Korea COPD subgroup Study Team cohort. J Korean Med Sci. 2016;31(4):553-560. 
International Journal of COPD

\section{Publish your work in this journal}

The International Journal of COPD is an international, peer-reviewed journal of therapeutics and pharmacology focusing on concise rapid reporting of clinical studies and reviews in COPD. Special focus is given to the pathophysiological processes underlying the disease, intervention programs, patient focused education, and self management protocols

\section{Dovepress}

This journal is indexed on PubMed Central, MedLine and CAS. The manuscript management system is completely online and includes a very quick and fair peer-review system, which is all easy to use. Visit $\mathrm{http}: / / \mathrm{www}$.dovepress.com/testimonials.php to read real quotes from published authors.

Submit your manuscript here: http://www.dovepress.com/international-journal-of-chronic-obstructive-pulmonary-disease-journal 